\title{
Percepção dos Profissionais para Implantação do Teste Rápido para HIV e Sífilis na Rede Cegonha
}

\author{
The Professional's Perception for the Implantation of the Rapid Test for HIV \\ and Syphilis in Rede Cegonha
}

\section{La Percepción de los Profesionales para la Implantación de la Prueba Rápida para VIH y Sífilis en la Rede Cegonha}

\author{
Rejane Rosaria Grecco dos Santos ${ }^{1}$ \\ Universidade Federal do Rio Grande (FURG) \\ Isadora Freire \\ Pontifícia Universidade Católica do Rio Grande do Sul (PUCRS) \\ Adolfo Pizzinato \\ Universidade Federal do Rio Grande do Sul (UFRGS) \\ Kátia Bones Rocha \\ Pontifícia Universidade Católica do Rio Grande do Sul (PUCRS)
}

\begin{abstract}
Resumo
O artigo investiga a avaliação dos profissionais da Atenção Primária (AP) sobre a implantação do aconselhamento e do teste rápido de HIV e Sífilis na Rede Cegonha (RC). Trata-se de um estudo qualitativo, descritivo e exploratório, no qual foram realizadas 13 entrevistas semiestruturadas com profissionais da AP, analisadas a partir da análise temática. Os resultados apontam a falta de conhecimento dos profissionais em relação às inovações da RC na AP. Os profissionais receberam capacitações referentes à testagem rápida, porém o matriciamento foi considerado inexistente. A solicitação do teste rápido das gestantes é realizada de forma compulsória. O aconselhamento, quando presente, é restrito ao pré-teste de HIV e outras Infecções Sexualmente Transmissíveis (IST), tendo caráter informativo, desconsiderando as especificidades da gestação. Indica-se a necessidade de se refletir sobre a autonomia das mulheres durante o pré-natal e o aconselhamento, espaço este que pode ser repensado como um momento de fortalecimento e acolhimento.

Palavras-chave: HIV/AIDS, teste rápido de HIV, aconselhamento, gestantes
\end{abstract}

\begin{abstract}
The article investigates the evaluation of the Primary Health Care (PHC) professionals about the implementation of the HIV and Syphilis rapid test in the care policy for pregnant women (CPPW). This is a qualitative, descriptive and exploratory study, which were conducted 13 semi-structured interviews with professionals, and analyzed from the thematic analysis. The results show a lack of knowledge of professionals about the innovations of CPPW in PHC. The professional received trainings for the rapid test, but the matricial support, with longitudinal supervision with specialized professionals, was considered inexistent. The test request of the pregnant women is released of compulsory form. Counseling is restricted to the pre-test of HIV and other Sexually Transmitted Infections (STIS), and just informative, disregarding the specifics of pregnancy. It is necessary to rethink the women's autonomy during the prenatal care and counseling and to construction these spaces as a moment of empowerment and welcoming. Keywords: HIV/AIDS, HIV rapid test, counselling, pregnant women
\end{abstract}

\section{Resumen}

El artículo investiga la evaluación de los profesionales de la Atención Primaria (AP) en relación a la implantación del asesoramiento y prueba rápida del VIH y Sífilis en la red de atención a las gestantes. El presente estudio es cualitativo, descriptivo y exploratorio, en el cual se realizaron 13 entrevitas semiestructuradas con los profesionales de la AP, analizadas a partir del análisis temático. Los resultados señalan la falta de conocimiento de los profesionales en relación a las innovaciones del RC en la AP. Los profesionales recibieron capacitaciones referentas a la prueba rápida del VIH, pero la supervisión longitudinal de los

\footnotetext{
${ }^{1}$ Endereço de contato: Fundação Universidade do Rio Grande, Carreiros, Rio Grande, RS, CEP: 96203-900. E-mail: rejanegrecco@ymail.com
} 
casos fue considerado inexistente. La solicitud de la prueba rápida de VIH y otras Infecciones Sexualmente Transmissibles (ISTS) a las gestantes se realiza de forma obligatoria. El asesoramiento, cuando presente, se limita al pre-test del VIH y otras ISTs, teniendo carácter informativo, desconsiderando las especificidades de la gestación. Se indica que es necesario repensar la autonomía de las mujeres durante el prenatal y el asesoramiento, para que este sea un espacio de empoderamiento y acogida para las mujeres.

Palabras clave: VIH/SIDA, prueba rápida del VIH, tomar consejo, embarazadas

\section{Introdução}

No Brasil (2016), os dados do Boletim Epidemiológico sobre a detecção de Síndrome da Imunodeficiência Adquirida (HIV/AIDS) revelam um quadro de estabilização nos últimos dez anos, com média de 20,7 casos para cada 100 mil habitantes. Em 2015, o Rio Grande do Sul foi o estado com maior taxa de deteç̧ão do país, chegando a 34,7 casos para cada 100 mil habitantes, e Porto Alegre foi a capital com maior taxa de casos, com 70 para cada 100 mil habitantes, sendo quase três vezes maior que a média do Brasil. Por outro lado, a taxa de detecção de HIV entre gestantes apresentou uma tendência de aumento nos últimos dez anos no Brasil, no entanto, o Rio Grande do Sul (10,1 casos/mil nascidos vivos) e Porto Alegre (22,9 casos/ mil nascidos vivos) continuam em primeiro lugar no ranking de deteç̧ão de HIV em gestantes (Brasil, 2016).

Desde o ano 2000, a faixa etária entre 20 a 24 anos é a que apresenta o maior número de casos de gestantes infectadas pelo HIV notificados (28,6\%). Frente a esse quadro, a partir do mesmo ano, o Ministério da Saúde implantou a política de descentralização do aconseIhamento e do teste rápido de HIV e outras Infecções Sexualmente Transmissíveis (IST) para atenção primária (Brasil, 2003).

Em 2011, o Ministério da Saúde institui, no âmbito do Sistema Único de Saúde (SUS), a Rede Cegonha (RC), que configura uma rede de cuidados visando assegurar à mulher o direito ao planejamento reprodutivo e à atenção integral na gravidez, parto e puerpério, com o objetivo de reduzir a mortalidade materna e infantil, através da ampliação e qualificação das práticas na Atenção Primária (Brasil, 2011). Em 2012, o teste rápido para HIV, Sífilis e outras Hepatites foi incluído no âmbito da RC, sendo Porto Alegre a capital pioneira nesse processo, contribuindo para o diagnóstico no pré-natal, sendo esse uma ferramenta tecnologia fundamental para a redução da transmissão vertical (Brasil, 2012).

Cabe destacar que a atenção à saúde sexual e reprodutiva é uma das áreas de atuação prioritárias da Atenção Primária (AP) à saúde. Deve ser ofertada observando-se como princípio o respeito aos direitos sexuais e aos direitos reprodutivos (Brasil, 2013). Neste sentido, a AP - por ser a porta de entrada dos usuários no SUS - ao oferecer o teste rápido e o aconselhamento para HIV, sífilis e hepatites, tem um papel essencial na prevenção de IST (Souza \& Freitas, 2012).

De acordo com o Ministério da Saúde (Brasil, 1998), o aconselhamento tem como objetivo reduzir o estresse do usuário que chega para realizar a testagem e propiciar a reflexão sobre riscos e adoção de práticas sexuais mais seguras ou planejadas, buscando uma maior adesão ao tratamento e contribuindo para uma melhor comunicação entre parceiros sexuais. Do mesmo modo, propõe o resgate da integralidade do cuidado ao usuário, o qual é percebido como sujeito ativo em suas ações de saúde, implicando o reconhecimento de sua subjetividade e autonomia (Brasil, 1998). Carvalho, Both, Alnoch, Conz, \& Rocha (2016) destacam a importância de o aconselhamento considerar a singularidade dos usuários, bem como suas experiências e estratégias frente às IST. 
No contexto de descentralização do teste rápido e aconselhamento, além das capacitações para a realização do Teste Rápido de HIV, Sífilis e outras hepatites e do aconselhamento, os profissionais devem receber o apoio matricial. Esse apoio é baseado na troca de saberes entre a equipe matricial (composta por especialistas) e a equipe da AP (Campos \& Domitti, 2007) no sentido de qualificar o atendimento aos usuários.

Cabe ressaltar que, apesar dos profissionais da AP reconhecerem a importância do aconselhamento pré e pós-teste como ferramentas de cuidado, nem sempre este é realizado em nível primário (Araújo, Vieira, \& Araújo, 2009; Araújo, Vieira, \& Galvão, 2011). Assim como a territorialização, que é um dos pilares da AP, e pode facilitar o acesso das pessoas ao teste e ao aconselhamento, pode também acentuar as problemáticas associadas ao sigilo das informações, privacidade dos usuários e estigmas associados ao HIV/AIDS (Seoane \& Fortes, 2009; Zambenedetti \& Both, 2013), dificultando a acessibilidade dos usuários aos serviços de saúde.

Neste contexto, Porto Alegre apresenta dados alarmantes referentes à HIV/AIDS e principalmente à infecção das gestantes, estando em primeiro lugar nos rankings de detecção, sendo 8,4 vezes maior que a taxa nacional e 2,3 vezes maior que a taxa do Rio Grande Sul. Além disso, por ter sido a primeira cidade do Brasil a descentralizar o aconselhamento e 0 teste rápido para a $\mathrm{AP}$, este ainda vem sendo aprimorado e adequado às rotinas dos serviços de saúde, tornando-se ferramenta fundamental nesses espaços. Portanto, o objetivo deste estudo foi investigar a avaliação que os profissionais da AP em saúde fazem sobre a implantação do aconselhamento e do teste rápido de HIV, Sífilis e hepatites virais na RC durante o pré-natal, no município de Porto Alegre, RS.

\section{Método}

Estudo de abordagem qualitativa, descritivo e exploratório. A primeira etapa do processo de seleção dos participantes foi orientada pela distribuição territorial dos serviços de saúde de Porto Alegre. A cidade conta com 123 serviços de saúde, distribuídos em oito gerências distritais, e, a partir disso, foram selecionados os serviços que mais testam e os que menos testam de cada gerência. Por meio dos critérios de seleção - ter no mínimo um ano de experiência na AP e realizar o teste rápido e o aconselhamento no pré-natal -, foram entrevistados 13 profissionais pertencentes a 11 serviços, totalizando cinco Estratégias Saúde da Família (ESF) e seis Unidades Básicas de Saúde (UBS). Em cada um dos serviços escolhidos, houve um contato prévio para apresentação da pesquisa, com vista a convidar os trabaIhadores que se encaixavam nos critérios para participar. Em alguns serviços, mais de um trabalhador assinalou o desejo de participar da pesquisa. Assim, participaram do estudo: um médico clínico geral, uma médica ginecologista, dez enfermeiras e um enfermeiro.

O tempo médio de atuação na AP dos profissionais entrevistados foi de 10 anos e 7 meses, compreendendo profissionais que estão há apenas 2 anos até 20 anos. Em relação ao tempo que atuam realizando o teste rápido, identificou-se desde um ano até dez anos, com média de cinco anos. As idades dos participantes variam entre 29 e 60 anos, com média de 37 anos e 5 meses. Os participantes foram identificados de acordo com a sua profissão e com a data da entrevista.

O instrumento utilizado para a coleta de dados foi uma ficha de dados com questões fechadas, que compreenderam informações referentes a idade, sexo, tempo de atuação na AP 
e experiência com o teste rápido. A entrevista semiestruturada estava composta por questões que visaram identificar conhecimentos acerca dos testes rápido de HIV, hepatites virais e sífilis. Além disso, foram realizadas perguntas sobre a descentralização dos testes para a AP, assim como sobre a capacitação e matriciamento. Em relação à implantação do teste no pré-natal, foram investigadas as percepções dos profissionais sobre os benefícios da política de descentralização para a gestante, bem como a reação delas ao receberem a indicação para realizar o teste rápido, facilidades e dificuldades em solicitar o teste rápido. Também foram abordadas questões referentes a preparação dos profissionais para a realização do teste rápido, facilidades e dificuldades para realizar o aconselhamento, conhecimentos acerca da RC e percepções sobre as mudanças com a implantação da RC na AP.

As entrevistas foram realizadas entre os meses de maio e junho de 2015. Estas tiveram duração média de 45 minutos. Após a coleta, as entrevistas foram transcritas e codificadas com o apoio do software Atlas.ti. Para a análise dos dados foi utilizada a Análise Temática (AT) (Braun \& Clarke, 2006) a partir dos seguintes passos: transcrição cuidadosa das entrevistas; codificação inicial dos temas que surgiram como mais relevantes a partir da exploração de todo o material, os quais foram considerados como coerentes, consistentes e distintos entre si. Depois de escolhidos os temas, as entrevistas foram relidas e recodificadas, os dados das entrevistas foram interpretados e organizados a partir da análise que buscou considerar um bom equilíbrio entre a narrativa analítica e extratos ilustrativos que são fornecidos no texto. Desta forma, uma pesquisadora inicialmente codificou as entrevistas utilizando o Atlas. ti, e outros dois juízes analisaram as entrevistas de forma independente, contribuindo com possíveis codificações complementares. As discrepâncias das codificações foram discutidas entre todos os pesquisadores/juízes e, por fim, foi estabelecido um consenso para realizar a construção dos eixos temáticos mais relevantes.

O presente estudo foi financiado pelo Conselho Nacional de Desenvolvimento Científico e Tecnológico (CNPq) (Edital Universal n. 482051/2013-2) e aprovado pelo Comitê de Ética em Pesquisa da Pontifícia Universidade Católica do Rio Grande do Sul e da Secretaria Municipal de Saúde de Porto Alegre, via Plataforma Brasil (n. 779.500). Este estudo foi realizado respeitando e atendendo às diretrizes da Comissão Nacional de Ética em Pesquisa (CONEP) através da Resolução n. 466/12, do Conselho Nacional de Saúde (CNS). Para a inclusão no estudo, foi solicitado aos participantes que assinassem o Termo de Consentimento Livre e Esclarecido (TCLE), a fim de garantir a liberdade de participação nas entrevistas, a privacidade e o sigilo das informações recebidas.

\section{Resultados e Discussão}

A partir das análises realizadas, os resultados da pesquisa foram divididos em seis eixos temáticos: 1) Conhecimento e avaliação da política; 2) Capacitação/matriciamento; 3) Solicitação do teste; 4) Aconselhamento pré e pós-teste de gestantes; 5) Abordagem da Sexualidade e 6) Inclusão do parceiro.

\section{Conhecimento e Avaliação da Política}

Neste eixo foi analisado o que os profissionais afirmam saber sobre a RC, considerando de que modo eles se apropriam e avaliam esta rede de cuidados. Por meio das análises das 
entrevistas, foi possível identificar que apenas três entrevistados conhecem a fundo a RC, já os outros dez entrevistados relatam conhecer pouco ou quase nada, apontando para a falta de apropriação dos conceitos relacionados à implantação da política, como ilustra a fala a seguir:

Vou te dizer que agora eu não lembro o que é a $R C$, mas eu sei que tem a ver com gestantes e com os bebês. Agora como é o fluxo, como é que foi implantado, eu realmente não sei (Enfermeira, comunicação pessoal, 04/05/2015).

É importante que os profissionais atuantes na AP saibam quais são as ações que a RC prevê, pois estas visam a melhoria do acesso e da qualidade da assistência à mulher e à criança, e se dão principalmente por meio da vinculação entre usuário e profissional. No entanto, nem sempre as demandas são plenamente integradas às rotinas das equipes (Cavalcanti, Gurgel, Vasconcelos, \& Guerrero, 2013) contribuindo para o desconhecimento sobre as diretrizes das políticas implantadas em nível primário.

Cabe destacar que três profissionais referem que as inovações em nível primário em relação à RC estão bastante restritas à implantação do teste rápido. Além disso, outros sete profissionais relatam não reconhecer inovações em nível primário referente à RC. Segue um relato de cada, respectivamente:

Eu não vejo muita novidade, porque essas coisas preconizadas na $R C$ na verdade já são feitas, só que aí é mais intensificado na verdade, mas isso sempre foi feito. Não era feito o teste rápido porque até então não existia, mas era feito exame de laboratório (Enfermeira, comunicação pessoal, 18/05/2015).

Nada, não tem nada de diferente para nós com ou sem a RC. Talvez lá a nível de maternidade, mas para nós, não interfere em nada, nem para melhor, nem para piorar (Enfermeira, comunicação pessoal, 20/05/2015).

As entrevistas, portanto, apontam para a falta de mudanças com a política em nível primário, sendo estas restritas somente aos hospitais. Neste sentido, é apontada a necessidade de modificações transversais aos mais diferentes níveis de atenção.

\section{Capacitação/Matriciamento}

Em relação às capacitações para realização do aconselhamento e do teste rápido para identificação de infecção por HIV e outras IST dentro da RC, todos os profissionais referiram ter participado de treinamentos. No entanto, oito relataram que consideraram a capacitação insuficiente, como no relato a seguir:

Faltou mais horas de capacitação, seria interessante para abordar as diferentes formas. Esses casos de relação extraconjugal, que acontece, são pouco trabalhados, é falado que 'ah, promiscuidade', mas qual é o conceito que tu vais dar para os profissionais da rede básica, defina promiscuidade (Médica Ginecologista, comunicação pessoal, 04/05/2015).

Percebeu-se que os profissionais não se sentem totalmente preparados para a realização do aconselhamento, visto que este não considera as inúmeras situações diárias que chegam aos serviços de saúde. Quando abordado especificamente o matriciamento, apenas um pro- 
fissional referiu ter participado ativamente dessa prática, mas que esta não se configura como uma prática com acompanhamento longitudinal de equipes especializadas aos serviços de saúde. Os demais profissionais, inclusive, desconheciam a existência de tal estratégia na RC.

Matriciamento hoje em dia só existe por telefone, ou eventualmente tem umas novas reciclagens, capacitação (Enfermeira, comunicação pessoal, 14/10/2015).

Um estudo com os profissionais que realizaram o matriciamento das equipes de atenção primária de Porto Alegre apontou que em apenas em um dos oito distritos de saúde da cidade o matriciamento é atualmente ativo (Rocha, Santos, Conz, \& Silveira, 2016). Neste contexto, questiona-se como acontece o acompanhamento das equipes e o impacto que isso pode causar nas ações de descentralização do teste rápido e aconselhamento.

\section{Solicitação do Teste}

Outro aspecto investigado foi o processo de solicitação do teste para as gestantes. Neste aspecto todos os entrevistados referem que a solicitação do teste faz parte de um protocolo e de uma rotina do serviço como referido a seguir:

No primeiro trimestre, na primeira consulta a gente faz, é o protocolo da consulta, então ela já sai daqui fazendo o teste, não é perguntado se vai fazer ou não (risos), é a rotina da consulta (Médica Ginecologista, comunicação pessoal, 04/05/2015).

Faz parte, a gente não perguntar, a gente nunca pergunta para elas, elas chegam e a gente faz, eu nunca perguntei (Enfermeira, comunicação pessoal, 18/05/2015).

A forma protocolar como é realizado o teste dentro do pré-natal permite que se reflita a autonomia das usuárias dentro da rede de cuidados da RC, se seus direitos de usuárias do sistema estão sendo ignorados em detrimento das expectativas de saúde do seu bebê, e a mulher assume então o papel de corpo reprodutivo. Estudos anteriores indicam que o teste de HIV costuma ser realizado nos serviços de saúde de forma compulsória, tornando irrelevante a autonomia das gestantes (Fonseca \& Iriart, 2012). Os autores também referem que, comumente, o aconselhamento se limita a informar o diagnóstico e dar orientações somente àquelas pessoas cujos resultados foram positivos e, dessa forma, desconsiderando a agência das usuárias em tomarem por si decisões no tocante à sua saúde.

A autonomia das mulheres dentro dos espaços de saúde é um dos tópicos principais de discussão e análise dentro do campo da bioética feminista (Diniz \& Guilhem, 2009). É urgente a necessidade de desnaturalizar prescrições sob o corpo em relação aos significados sociais atribuídos a sua existência, e essas discussões se dirigem à compreensão do gênero como um aspecto fundamental para a interpretação do próprio conceito de saúde. A forma impositiva com a qual o teste vem sendo realizado no pré-natal nos permite pensar em situações nas quais opere a coerção da vontade, principalmente neste contexto da gestação, no qual o seu corpo pode ser ainda mais objetificado pelas intervenções em saúde (Passos, Oliveira, Gomes Junior, \& Silva, 2013).

Um dos fatores que justificam a forma compulsória com que o teste é realizado relaciona-se ao discurso sanitário em prol da proteção ao recém-nascido e seu papel nas políticas de saúde materno infantil, como uma das entrevistadas referiu. 
É um teste que tem que fazer, é obrigado a fazer no pré-natal, para eu saber, para o nenê ficar bem. Isso é o motivo dela fazer o teste (Enfermeira, comunicação pessoal, 20/05/2015)

Ainda que o quadro de feminilização da AIDS corrobore para o aumento na demanda desse teste, um número cada vez maior de mulheres terá seus direitos de autonomia e de decisão desrespeitados, caso não se opere uma mudança de atitude de cuidado (Silva, Guilhem, \& Bampi, 2012).

Apenas um dos profissionais entrevistados expôs uma crítica à visão "formatada" na atenção realizada às gestantes.

Porque tudo é meio formatado, é mais difícil elas se negarem porque tem essa questão do

bebê, então a gente vai por essa linha (Enfermeiro, comunicação pessoal, 20/05/2015).

A possibilidade de refletir sobre os processos de trabalho pode propiciar que os profissionais consigam pensar sobre suas rotinas de trabalho e os seus desdobramentos na atenção oferecida, especialmente para que tenham seus direitos e sua dignidade respeitados e, também, que sejam capacitadas a enfrentar um possível resultado positivo no teste de HIV (Brasil, 2013).

\section{Aconselhamento Pré e Pós-Teste de Gestantes}

O aconselhamento, por se tratar de uma importante estratégia na implantação do teste rápido nas UBS, apresenta um papel chave dentro do pré-natal, como espaço de tomada de decisão conjunta, entre os profissionais e os usuários envolvidos (Silva, Guilhem, \& Bampi, 2012). Os profissionais entrevistados reconhecem o aconselhamento como um instrumento importante na realização do teste rápido, salientando a importância de considerar a integralidade do usuário que está ali, como aparece a seguir:

Tu tens que partir da realidade da pessoa e tentar que na realidade, com as crenças dela, que ela se proteja da melhor maneira possível (Médica Ginecologista, comunicação pessoal, 04/05/2015).

Em relação à realização do aconselhamento, doze profissionais referiram que ele é feito e apenas um referiu que não realiza.

Vou ser sincero, na prática pouquíssimos médicos fazem, poucos, acho que quase nenhum, eu mesmo vou falar, não é minha rotina fazer. Faço se for preciso, mas não é uma coisa que eu pus na agenda para fazer porque requer tempo e tem as outras demandas (Enfermeira, comunicação pessoal, 04/05/2015).

Essa fala evidencia a dificuldade de alguns profissionais realizarem o aconselhamento, tanto o pré-teste quanto o pós-teste, fato que já foi apresentado em outros estudos, que enfatizam uma não realização deste no contexto da atenção primária (Araújo, Vieira, \& Galvão, 2011; Seoane \& Fortes, 2009).

Os profissionais também foram questionados sobre como o aconselhamento era realizado. A maioria referiu que no aconselhamento pré-teste abordam as questões de relacionamentos sexuais (parceiro fixo ou não), história familiar, o que é HIV/AIDS, como ocorre a transmissão, como se dá a prevenção, a importância do uso de preservativo, comportamentos de 
risco e o que seria a janela imunológica. No entanto, dos treze profissionais entrevistados, oito referem que o aconselhamento pré-teste no pré-natal é o mesmo dos outros usuários que frequentam o serviço, desconsiderando as questões gestacionais, como aparece nesta fala:

O pacote básico é o mesmo, isso varia um pouco do entendimento de cada um, se tu vais fazer uma abordagem diferenciada, para uma para outra (Enfermeira, comunicação pessoal, 18/05/2015).

O pacote básico referido está associado aos aspectos mais informativos em relação à doença e ao teste. Neste contexto, os aspectos mais associados ao apoio emocional e à gestão de riscos (Brasil, 1998; Carvalho et al., 2016) parecem não ser contemplados. Além disso, as particularidades da gestação não parecem ser abordadas, fato que deveria ser melhor trabalhado pelos profissionais.

É necessário atentar que, como é apresentado na literatura, o pós-aconselhamento, enquanto estratégica de cuidado, é confundido com apenas informar o diagnóstico e dar orientações pós-teste somente para aquelas pessoas cujos resultados foram positivos (Diniz \& Guilhem, 2009). Neste sentido, dos treze entrevistados, apenas um profissional destacou a importância do pós-teste independente do resultado do teste. Foi possível perceber que a abordagem vai diferenciar-se apenas se, após o exame, o resultado for positivo, como referido a seguir:

É importante ter o tratamento mais humanizado, dar todos os encaminhamentos quando positivo (Enfermeira, comunicação pessoal, 18/05/2015).

Assim, não há uma reflexão sobre o resultado e sobre as práticas sexuais que a levaram até ali. O aconselhamento pós-teste pode ser um importante momento para pensar as estratégias de prevenção e autocuidado do usuário. Os profissionais ainda apontam o aconselhamento como um desafio da política de descentralização do teste rápido para a AP, pois relatam não terem tido um espaço adequado durante as capacitações para isso.

É a gente nunca teve uma capacitação específica para o aconselhamento, entendeu?! É uma coisa interessante. A gente tem sobre o teste em geral, ai se fala do aconselhamento. Mas específico do aconselhamento a gente nunca teve. Eu acho que seria importante, até por várias dúvidas que surgiram agora (Enfermeira, comunicação pessoal, 26/05/2015).

Faz-se necessário atentar para esta etapa do processo de descentralização do teste rápido especificamente com as demandas da RC, pois as equipes demonstram não estarem totalmente organizadas para a realização do aconselhamento, principalmente considerando as especificidades da gestação.

A realização de testes para diagnóstico de IST, acompanhado do aconselhamento, representa uma importante estratégia de cuidados perinatais e minimiza a vulnerabilidade que envolve a gestante e a criança expostas ao vírus (Silva, Guilhem, \& Bampi, 2012). A escuta qualificada facilita o vínculo entre a equipe de saúde e a mulher. Trata-se de um momento para identificar e minimizar conflitos e situações que ocasionam fragilidades. O profissional pode avaliar vulnerabilidades relacionadas ao HIV e a outras esferas da vida da gestante, como a possibilidade de contrair infecções e a preparação para o diagnóstico. 


\section{Abordagem da Sexualidade}

Em geral, os profissionais de saúde sentem dificuldades de abordar os aspectos relacionados à saúde sexual. Trata-se de uma questão que levanta polêmicas, na medida em que a compreensão da sexualidade está marcada por preconceitos e tabus (Brasil, 2013). Fato que foi observado nas entrevistas realizadas, como por exemplo:

A gente foca na prevenção, da transmissão e do cuidado que ela tem que ter, mas não muito aprofundado do cuidado sexual (Enfermeira, comunicação pessoal, 18/05/2015).

Nesta direção, alguns entrevistados referem centrar a atenção do aconselhamento no uso ou não de preservativos durante a gestação.

Geralmente elas falam, ah agora eu estou com o meu marido, mas há anos eu não tinha parceiro fixo, eu ia para a festa, transava com um, transava com outro. Porque sempre quando a gente conversa, surge alguma dúvida, daí a gente vai explicando, olha, às vezes tem muitas doenças sexualmente transmissiveis, algumas contam mais, algumas contam menos, outras não contam nada (Enfermeira, comunicação pessoal, 20/05/2015).

A partir do referido e das entrevistas se observa que a própria forma com a qual eles falam sobre o tema já mostra a dificuldade de nomear, explicitar, quais aspectos da sexualidade poderiam ser trabalhados. Por outro lado, o profissional de saúde, ao considerar a sexualidade imersa na gestação, compreenderá melhor a gestante e com isso terá a oportunidade de melhor articular com ela a gestação e suas implicações na vida sexual (Camacho, Vargens, \& Progianti, 2010).

O tabu associado às questões da sexualidade aparece na fala dos entrevistados, que referem uma dificuldade também das usuárias de falarem sobre o tema:

Vai muito de uma avaliação que eu faço, nem sempre eu pergunto ... A maioria acha estranha a pergunta, parece o que eu tenho a ver com isso? Embora eu tente o máximo não fazer em tom de julgamento, muitas franzem a testa, ficam meio desconcertadas, mas acabam falando. Mas acho que rola um constrangimento às vezes, mesmo deixando bem à vontade a pessoa (Enfermeira, comunicação pessoal, 26/05/2015).

Neste sentido, estudos confirmam a fragilidade das orientações sobre sexualidade nas consultas de pré-natal e reforçam que os profissionais de saúde devem trabalhar questões relativas à sexualidade no período gestacional (Viana, Barrêto, Fonseca, Costa, \& Soares, 2013). Por outro lado, um dos aspectos que é salientado por um profissional entrevistado é a importância do vínculo entre profissional e usuária, para que as temáticas associadas a sexualidade possam ser abordadas. Provavelmente, se existe um maior vínculo, uma maior abertura ao diálogo e uma atenção singular, abordar temas relativos à sexualidade pode ser uma tarefa mais fácil para ambas as partes.

\section{Inclusão do Parceiro}

As ações voltadas para a saúde sexual, em sua maioria, têm sido focadas apenas na saúde reprodutiva, tendo como alvo a mulher adulta (Brasil, 2013). Essa situação reflete-se na baixa inclusão do parceiro durante o pré-natal. Os entrevistados afirmam que os parceiros, 
apesar de serem convidados por suas parceiras a vir realizar o teste e participar do pré-natal, raramente aparecem. Apenas um profissional disse que alguns parceiros participam, os outros doze profissionais afirmaram que a adesão por parte dos parceiros é quase nula, referindo que essa dificuldade pode estar associada aos papéis de gênero estereotipados e o funcionamento dos serviços:

Pela resistência dos homens mesmo né, é questão cultural né, não sei se é um pouco de medo... Muitos falam que não tem tempo de vir no posto, tem mais resistência mesmo (Enfermeira, comunicação pessoal, 04/05/2015).

Alegam a questão do trabalho e tal, daí eu falo, olha eu posso fornecer uma declaração, um comprovante de comparecimento (Enfermeira, comunicação pessoal, 26/05/2015).

As construções sociais de gênero corroboram para o afastamento das questões relativas ao autocuidado de saúde do homem (Silva \& Brito, 2010). Os atributos relacionados à masculinidade tradicional potencializam desigualdades sociais, invisibilizando necessidades e demandas dos homens e reforçando o estereótipo de que os serviços da atenção primária são espaços feminilizados (Couto et al., 2010).

O trabalho aparece como aspecto que restringe o acesso e o uso dos serviços pelos homens. A forma e os horários de funcionamento dos serviços de saúde dificultam que estes sejam utilizados pelos homens. Além disso, não há respaldo legal que assegure a presença do parceiro no acompanhamento do pré-natal, visto não haver justificativa para falta dele ao trabalho (Silva \& Brito, 2010). Ainda, a própria RC e a política de saúde materno infantil centram a sua atenção na saúde da mulher e do bebê, o que mostra uma vulnerabilidade programática no que diz respeito à inserção dos homens em lógicas de cuidado.

Percebe-se, segundo as entrevistas, que os usuários homens que realizam o teste só o fazem se o resultado da mulher for positivo, o que pode ser confirmado pela fala da profissional:

Os que dão negativo, eles não vêm, claro, a gente convida, porque é importante eles fazerem também. Agora se deu positivo, eles são praticamente convocados a vir, principalmente pela gestante, porque tem uma criança envolvida (Enfermeira, comunicação pessoal, 26/05/2015).

Portanto, é preciso ampliar a abordagem para outras dimensões que contemplem a saúde sexual em diferentes momentos do ciclo de vida e também para promover o efetivo envolvimento e corresponsabilidade dos homens durante o pré-natal (Brasil, 2013).

\section{Considerações Finais}

Os resultados apresentados nesta pesquisa podem servir como um disparador para o desenvolvimento de novos trabalhos relacionados à inserção do teste rápido de HIV e IST e o aconselhamento no pré-natal em outras cidades do país. Assim, a partir deste estudo pôde-se identificar que há falta de apropriação em relação à política de implantação do teste rápido para HIV e Sífilis na RC. Além disso, cabe destacar que os profissionais referem que as inovações associadas à RC, em nível primário, estão restritas à descentralização do teste rápido. Esse aspecto reflete o quanto a ideia de uma rede de cuidados para garantir um melhor acolhimento e uma atenção integral à gestante e ao bebê, em todos os níveis de 
complexidade do sistema, deve ser repensada, pois ainda parece estar restrita aos hospitais e às testagens.

Os profissionais referiram ter recebido treinamento para realização do teste rápido e aconselhamento. Porém, destacam que não receberam uma capacitação para o aconseIhamento que considerasse as especificidades da gestação considerando, assim, as capacitações deficitárias. Outro aspecto destacado foi a ausência do matriciamento em nível primário, pois este está restrito a ligações telefônicas. É necessário atentar ao matriciamento, que é um dispositivo muito importante para qualificar tanto a realização do teste quanto as dificuldades associadas ao aconselhamento e aos resultados positivos. Diferentemente das capacitações, esse dispositivo configura-se como um acompanhamento longitudinal das equipes e que, de forma continuada, pode ser um espaço importante para problematizar questões associadas à abordagem da sexualidade que vêm sendo negligenciadas no espaço do pré-natal.

Cabe destacar que os profissionais referiram realizar o aconselhamento pré-teste, o que pode ser destacado como um aspecto positivo da implantação do teste em nível primário. No entanto, o aconselhamento pós-teste deve ser potencializado em termos de prevenção e promoção de saúde, visto que ainda há dificuldades de efetivação, sendo realizado apenas quando o resultado da testagem é positivo. Neste sentido, esforços necessitam ser feitos para qualificar o aconselhamento e principalmente direciona-lo às especificidades do pré-natal e singularidades das gestantes e dos seus companheiros.

O pré-natal pode se constituir em um espaço no qual a mulher pode exercer sua autonomia e cidadania, porém, a partir do relato dos profissionais, este parece estar, muitas vezes, reforçando lógicas de opressões as quais as mulheres já sofrem em função das relações assimétricas de gênero construídas na sociedade. Portanto, um dos desafios é pensar o espaço do pré-natal como um momento de fortalecimento das mulheres, de acolhimento, no qual essas possam expor suas dúvidas, seus medos e fortalecerem-se enquanto sujeitos de direitos.

Ao analisar as questões de gênero a partir de uma perspectiva relacional, é importante destacar a importância de serem desenvolvidos mecanismos para que os homens consigam também se envolver no pré-natal, desconstruindo estereótipos arraigados socialmente de que a gestação diz respeito somente às mulheres e que os homens não precisam participar desse processo, deixando assim de acessar os serviços de saúde. E para que isso se viabilize, existe a necessidade de esforços políticos no sentido de incluir o homem nas políticas de saúde em geral e, especificamente, na política materno-infantil, bem como que os profissionais percebam a importância deles no processo de atenção à saúde.

Em relação às limitações deste estudo, cabe destacar que se trata de um estudo qualitativo, no qual foram entrevistados alguns profissionais, que nos propiciaram através dos seus relatos de experiência pensar questões para qualificar a atenção no pré-natal, a partir do aconselhamento e do teste rápido. Futuros estudos quantitativos podem fazer um levantamento em toda a rede para avaliar em que medida o teste rápido de HIV e de outras IST vem sendo realizado, bem como avaliar mais sistematicamente o aconselhamento realizado na rede. 


\section{Referências}

Araújo, M. A. L, Vieira, N. F. C., \& Araújo, C. L. F. (2009). Aconselhamento coletivo pré-teste anti-hiv no pré-natal: Uma análise sob a ótica dos profissionais de saúde. Revista Baiana de Saúde Pública, 33(2), 122-135.

Araújo, M. A. L, Vieira, N. F. C., \& Galvão, M. T. G. (2011). Aconselhamento pré e pós-teste anti HIV em gestantes em Fortaleza, Ceará. Revista Espaço para a Saúde, 12(2), 18-27.

Brasil. (1998). Ministério da Saúde. Coordenação Nacional de DST e AIDS. Aconselhamento em DST, HIV e AIDS: Diretrizes e procedimentos básicos (2a ed.). Brasília, DF: Ministério da Saúde. 21p.

Brasil. (2003). Ministério da Saúde. Aconselhamento em DST/HIV/AIDS para a Atenção Básica. Brasília, DF: Ministério da Saúde. 30p. Disponível em http://bvsms.saude.gov.br/ bvs/publicacoes/manual_simplificado.pdf

Brasil. (2011). Ministério da Saúde. Portaria n. 1.459, de 24 de junho de 2011. Institui no âmbito do Sistema Único de Saúde - SUS - a Rede Cegonha. Brasília, DF: Ministério da Saúde.

Brasil. (2012). Ministério da Saúde. Departamento de Atenção Básica. Realização do Teste Rápido para HIV e Sífilis na Atenção Básica e Aconselhamento em DST/AIDS no âmbito da Rede Cegonha. Brasília, DF: Ministério da Saúde. 100p.

Brasil. (2013). Ministério da Saúde Secretaria de Atenção à Saúde. Departamento de Atenção Básica. Cadernos de Atenção Básica: Saúde sexual e saúde reprodutiva n. 26. Brasília, DF: Ministério da Saúde. 300p.

Brasil. (2016). Ministério da Saúde. Secretaria de Vigilância em Saúde. Boletim Epidemiológico: AIDS e DST. Brasília, DF: Ministério da Saúde. 64p.

Braun, V., \& Clarke, V. (2006). Using thematic analysis in psychology. Qualitative Research in Psychology, 3, 77-101.

Camacho, K. G., Vargens, O. M. C., \& Progianti, J. M. (2010). Adaptando-se à nova realidade: A mulher grávida e o exercício de sua sexualidade. Revista de Enfermagem UERJ, 18(1), 32-37.

Campos, G. W. S., \& Domitti, A. C. (2007). Apoio matricial e equipe de referência: Uma metodologia para a gestão do trabalho interdisciplinar em saúde. Cadernos de Saúde Pública, 23(2), 399-407.

Carvalho, F. T., Both, N. S., Alnoch, E. M., Conz, J., \& Rocha, K. B. (2016). Counseling in STD/HIV/AIDS in the context of rapid test: Perception of users and health professionals at counseling and testing centre in Porto Alegre. Journal of Health Psychology, 21, 379-389.

Cavalcanti, P. C. S., Gurgel, J. G. D., Vasconcelos, A. L. R., \& Guerrero, A. V. P. (2013). Um modelo lógico da Rede Cegonha. Physis: Revista de Saúde Coletiva, 23(4), 1297-1316.

Couto, M. T., Pinheiro, T. F., Valença, O., Machin, R., Silva, G. S. N., Gomes, R., Schraiber, L. B., \& Figueiredo, W. S. (2010) O homem na atenção primária à saúde: discutindo (in) visibilidade a partir da perspectiva de gênero. Interface - Comunicação, Saúde, Educação, 14(33), 257-270.

Diniz, D., \& Guilhem, D. (2009). Bioética feminista: O resgate político do conceito de vulnerabilidade. Revista Bioética, 7(2), 182-188. 
Fonseca, P. D. L., \& Iriart, J. A. B. (2012). Aconselhamento em DST/AIDS às gestantes que realizaram o teste anti-HIV na admissão para o parto: Os sentidos de uma prática. Interface - Comunicação, Saúde, Educação, 16(41), 395-407.

Passos, S. C. S, Oliveira, M. I. C, Gomes Junior, S. C. S., \& Silva, K. S. (2013). Aconselhamento sobre o teste rápido anti-HIV em parturientes. Revista Brasileira de Epidemiologia, 16(2), 278-287.

Rocha, K. B, Santos, R. R. G, Conz, J., \& Silveira, A. C. T. (2016). Transversalizando a rede: O matriciamento na descentralização do aconselhamento e teste rápido para HIV, sífilis e hepatites. Saúde em Debate, 40(109), 22-33.

Seoane, A. F., \& Fortes, P. A. C. (2009). A percepção do usuário do Programa Saúde da Família sobre a privacidade e a confidencialidade de suas informações. Revista Saúde e Sociedade, 18(1), 42-49.

Silva, F. C. B., \& Brito, R. S. (2010). Percepção de gestantes acerca das atitudes do companheiro diante da sua ausência no pré-natal. Rev. Rene, 11(3), 95-102.

Silva, O., Guilhem, D., \& Bampi, L. N. S. (2012). Trinta minutos que mudam a vida: Teste Rápido Anti-HIV Diagnóstico para parturientes e acesso ao pré-natal. Enfermagem em Foco, 3(4), 211-215.

Souza, M. C. M., \& Freitas, M. I. D. F. (2012). Aconselhamento em HIV/AIDS: representações dos profissionais que atuam na atenção primária à saúde. Revista Mineira de Enfermagem, 16(1), 18-24.

Viana, D. F., Barrêto, A. J. R., Fonseca, E. N. R., Costa, C. B. A., \& Soares, M. J. G. O. (2013). Vivência da sexualidade feminina no período gestacional: À luz da história oral temática. Ciência, Cuidado e Saúde, 12(1), 88-95.

Zambenedetti, G., \& Both, N. S. (2013). A via que facilita é a mesma que dificulta: Estigma e atenção em HIV-Aids na estratégia saúde da família - ESF. Fractal: Revista de Psicologia, 25(1), 41-58.

Recebido: 09/12/2016

última revisão: 14/09/2017

Aceite final: 18/09/2017

\section{Sobre os autores:}

Rejane Rosaria Grecco dos Santos - Doutora em Pediatria e Saúde da Criança pela Pontifícia Universidade Católica do Rio Grande do Sul (PUCRS). Professora na Universidade Federal do Rio Grande (FURG). E-mail: rejanegrecco@ymail.com

Isadora Freire - Bacharel em Psicologia pela Pontifícia Universidade Católica do Rio Grande do Sul (PUCRS).E-mail: isadora.nf@gmail.com

Adolfo Pizzinato - Professor no Departamento de Psicologia do Desenvolvimento e da Personalidade do Instituto de Psicologia da Universidade Federal do Rio Grande do Sul (UFRGS). E-mail: adolfopizzinato@hotmail.com

Kátia Bones Rocha - Professora da Pontifícia Universidade Católica do Rio Grande do Sul (PUCRS). Pesquisadora no Programa de Pós-graduação em Psicologia da PUCRS e coordenadora do grupo de pesquisa Psicologia, Saúde e Comunidades (PSC-PUCRS). E-mail: katiabonesrocha@gmail.com 
